\title{
Image of the Month
}

\section{Small Intestinal Diverticulitis}

\author{
STIJN J. B. VAN WEYENBERG, ${ }^{*}$ CHRIS J. MULDER, ${ }^{*}$ and JAN HEIN T. M. VAN WAESBERGHE ${ }^{*}$ \\ *Department of Gastroenterology and Hepatology and ${ }^{\ddagger}$ Department of Radiology, VU University Medical Center, Amsterdam, The Netherlands
}
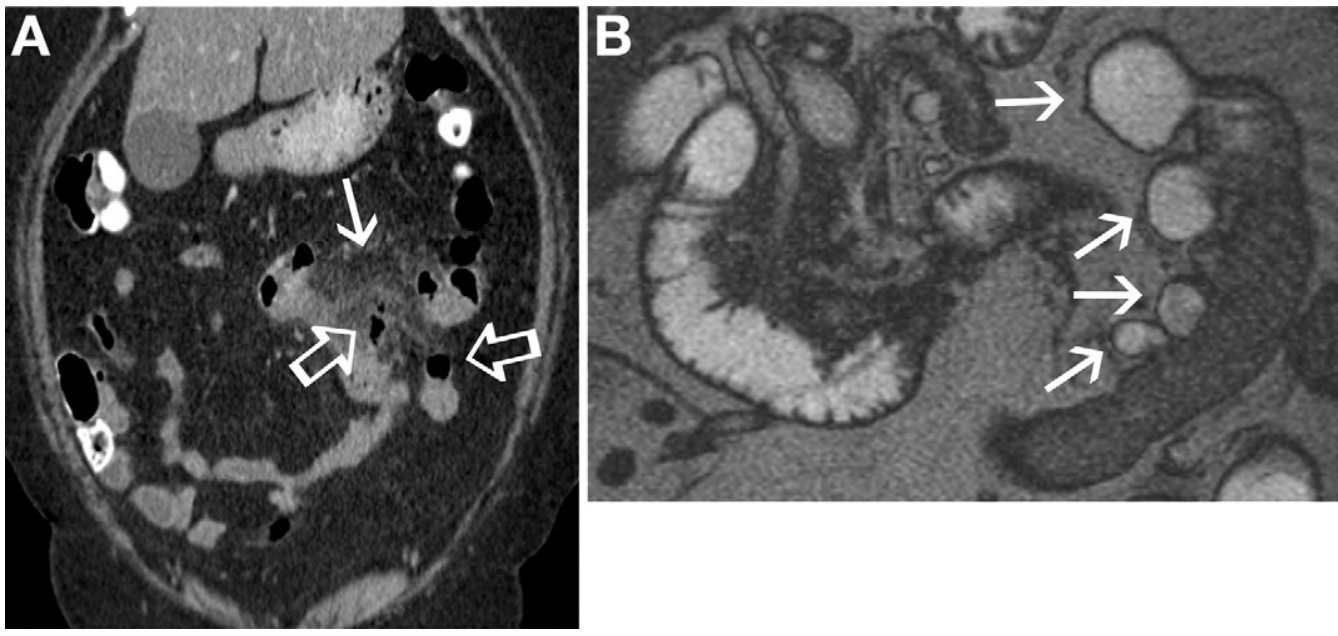

A 55-year old female patient was referred to our hospital because of severe abdominal pain, diarrhea, and fever. On physical examination she was febrile with a temperature of $103^{\circ} \mathrm{F}$ $\left(39.5^{\circ} \mathrm{C}\right)$ and had diffuse abdominal pain with rebound tenderness. Her laboratory studies showed C-reactive protein level of 248 $\mathrm{mg} / \mathrm{L}$. Contrast-enhanced abdominal computed tomography (CT) (Figure $A$ ) showed thickening of the proximal jejunal bowel wall, with infiltration of the mesenteric fat (arrow) and multiple extraluminal air bubbles surrounding this area (open arrows), some of which were possibly in contact with the small bowel lumen.

On the basis of these CT findings, jejunal diverticulitis with possible walled-off perforation was suspected, although other causes of small bowel perforation could not be excluded. Treatment with broad-spectrum antibiotics (ceftriaxone and metronidazole) was commenced, after which the patient's condition improved quickly. After 1 week of antibiotic treatment she was discharged. In 4 weeks C-reactive protein returned to normal values.

Seven weeks after presentation, magnetic resonance enteroclysis was performed to investigate the presence of jejunal diverticula and to define the regions of the small bowel affected (Figure $B$ ). Magnetic resonance enteroclysis showed multiple diverticula in the jejunum (arrows), but without small bowel wall thickening or mesenteric lymphadenopathy, confirming the initial diagnosis of jejunal diverticulitis.

The incidence of diverticula in the small intestine distal to the duodenum is reported to be between $0.06 \%$ and $2.3 \% .{ }^{1}$ Usually these diverticula are asymptomatic. Inflammation of jejunal diverticula presents in the same fashion as colonic diverticulitis. History taking and physical examination allow no discrimination between these 2 entities. Multiple radiologic imaging methods have been described in the diagnosis of small bowel diverticulitis., ${ }^{2,3}$ Small bowel radiography with oral barium or barium delivered by means of a jejunal catheter is able to visualize small bowel diverticula, but it usually does not provide information on mural, serosal, or mesenteric involvement. Although abdominal ultrasound is able to visualize extraluminal air and hyperechoic fat, it does not inform about the extent of small bowel diverticula. Conventional abdominal CT with oral contrast might allow a specific diagnosis of small bowel diverticulitis. However, in the absence of clearly depicted small bowel diverticula, as was the case in our patient, inflammation in and around small bowel loops cannot be distinguished from other conditions such as small bowel Crohn's disease and small bowel malignancies. Cross-sectional enteroclysis techniques are able to visualize not only the presence and extent of small bowel diverticula but also its inflammatory complications such as bowel wall thickening, mesenteric fat infiltration, and perforation.

\section{References}

1. Sibille A, Willocx R. Jejunal diverticulitis. Am J Gastroenterol 1992; 87:655-658.

2. Greenstein S, Jones B, Fishman EK, et al. Small-bowel diverticulitis: CT findings. AJR Am J Roentgenol 1986;147:271-274.

3. Kelekis AD, Poletti PA. Jejunal diverticulitis with localized perforation diagnosed by ultrasound: a case report. Eur Radiol 2002; 12(Suppl 3):S78-S81.

\section{Conflicts of interest}

The authors disclose no conflicts.

() 2010 by the AGA Institute

$1542-3565 / \$ 36.00$

doi:10.1016/j.cgh.2010.06.023 